\title{
La recherche participative en éducation et la transformation des pratiques au Saguenay-Lac-St-Jean : synthèse et de prospective
}

\author{
Loïc Pulido et Christine Couture, Université du Québec à \\ Chicoutimi
}

Il est de plus en plus reconnu que la recherche en éducation trouve davantage écho auprès des milieux de pratique si elle prend en considération l'écologie des milieux dans lesquels elle est menée, c'est-àdire ses éléments de contexte. II s'agit d'une prémisse mise en avant il y a déjà plusieurs années par les pionniers du courant de la recherche participative. Au Saguenay-Lac-St-Jean, ce courant a pris de l'ampleur dès la fin des années 1990 avec la création du Consortium régional de recherche en éducation (Minier; Courcy et Dumoulin). Depuis, une masse critique de chercheurs et d'intervenants des milieux scolaires de la région incarnent ce modus operandi en différents lieux de pratique, à propos de différents objets et à partir de différentes modalités de collaboration ainsi que de sources de financement. Afin de mieux saisir la richesse et la portée de ces travaux, ce collectif a présenté un échantillon de ce que des chercheurs, des étudiants de cycles supérieurs et des acteurs du milieu scolaire du Saguenay-Lac-St-Jean font ensemble pour transformer les pratiques et travailler collectivement à la réussite des élèves. La transformation des pratiques y est abordée sous différents angles. Elle peut mener à des ajustements en fonction d'un idéal à atteindre choisi collectivement (Savoie-Zajc, 2005), ou encore à des changements prescrits et attendus par les programmes dans un contexte de réforme (Lafortune, 2008). Entre ces deux pôles, plusieurs déclinaisons pouvant orienter différemment le travail de collaboration entre les acteurs sont possibles. À cet égard, la collaboration comme voie de transformation des pratiques peut aussi prendre des significations et des orientations 


\section{$\&$}

\section{REVUE HYBRIDE DE L'ÉDUCATION}

différentes. Dans une perspective de recherche, la collaboration suppose la co-construction de savoirs sur la pratique en partant de la prémisse que cela ne peut se faire qu'avec les enseignants (Desgagné, 1998). Mais la collaboration comme voie de transformation des pratiques ne se limite pas qu'à la recherche. Elle peut aussi s'inscrire dans un cadre professionnel (Marcel, Dupriez, et Bagnoud, 2007). C'est à toutes les variations possibles de ces postures que nous restons ouverts dans le regard transversal que nous portons sur les contributions de ce collectif pour éclairer la question de la transformation des pratiques par la voie de la collaboration. Ce regard transversal nous permet de réfléchir plus en profondeur aux rôles des acteurs qui travaillent ensemble pour la transformation des pratiques, aux savoirs mobilisés dans un tel processus de transformation, aux spécificités des options méthodologiques retenues par les acteurs, aux résultats de ces recherches, à leurs retombées ainsi qu'aux différents types de production et de diffusion qu'elles génèrent.

\section{Rôles}

L'attention portée au point de vue des acteurs, qu'il s'agisse d'enseignants, de parents, de stagiaires, de superviseurs, d'enseignants associés ou d'administrateurs, est perceptible dans chacun des exemples de recherche présenté dans ce collectif. Mais le rôle qui leur est attribué varie d'une contribution à l'autre. Considérés à titre d'acteurs compétents dans les contributions de Couture, Aurousseau, Lévesque et Tremblay, d'Amboulé-Abath, Larouche et Lessard, ainsi que de Duquette, Lauzon et Saint-Gelais, selon le modèle de la recherche collaborative (Desgagné, Bednarz, Couture, Poirier, et Lebuis, 2001), leur expertise professionnelle est mise de l'avant pour intégrer le point de vue de la pratique dans la coconstruction de nouveaux savoirs sur l'objet de la recherche. Coulombe, Doucet, Zourhlal et Thibeault accordent quant à eux une place importante à la notion de collaboration, mais davantage dans un cadre professionnel (Marcel, Dupriez, et Bagnoud, 2007). Cependant ce terme ne sert pas tant à définir le rôle de chacun qu'à exposer un niveau d'interrelation souhaitée entre les participants, ce qui apporte un éclairage différent. 


\section{REVUE HYBRIDE DE L'ÉDUCATION}

Lessard utilise le terme praticien-chercheur, en référence à la rechercheaction (Anadón et Savoie-Zajc, 2007), cette fois-ci pour qualifier le statut des acteurs des milieux de pratique. Dans ce travail, leur rôle consiste, à partir d'un portrait dressé par la chercheuse, à identifier des pistes d'actions possibles pour répondre aux objectifs d'une recherche-action. Dans ce cas, leurs compétences, en tant que professionnels, sont mises de l'avant pour alimenter la dimension praxéologique de la rechercheaction. Cody, Monney, Labrecque et Boisvert emploient également le terme collaborateur dans un cadre plus professionnel. Il est intéressant de noter que dans cette contribution, les tâches ne sont pas présentées comme réparties selon la provenance (milieu de recherche ou milieu de pratique). L'ensemble des acteurs est qualifié de collaborateurs, et l'ensemble s'empare de tous les objectifs.

En somme, ce collectif permet de constater que les recherches participatives présentées se distinguent sur plusieurs axes, dans leur manière de présenter les rôles des acteurs issus des milieux de pratique. D'une part, certaines répartissent les rôles, chacun se voyant dévolu une part de réflexion alors que d'autres constituent des équipes sur la base de compétences complémentaires, le groupe constitué ayant ensuite à sa charge l'avancée de l'ensemble du projet. D'autre part, lorsque les rôles sont répartis, certains travaux mettent l'accent sur l'importance des professionnels des milieux de pratique pour comprendre le contexte de la recherche, alors que d'autres mettent en lumière l'expertise professionnelle des praticiens. Nous pensons que le premier axe préoccupe toutes les recherches participatives. Nous voyons le second comme davantage contingent : les recherches « en classe » insistant sur les compétences en termes de savoir-faire des milieux de pratique et les recherches qui se font sur un grain plus «mezzo », au niveau d'un établissement par exemple, le mettant moins en avant.

En termes de prospectives et dans une perspective de partage des travaux issus des recherches participatives, la précision du rôle de tous les partenaires est essentielle pour faire comprendre comment s'est 


\section{$\&$}

\section{REVUE HYBRIDE DE L'ÉDUCATION}

réalisée la recherche. Des réflexions d'ordre conceptuel seraient à cet égard les bienvenues, car les notions existantes ne semblent pas rendre justement compte de la richesse des pratiques de recherche en la matière.

En termes de prospectives toujours, il serait intéressant d'expliciter davantage le rôle des chercheurs dans les recherches participatives. À première vue, cela peut paraitre aller de soi, mais seulement lorsque l'on est soi-même chercheur. Or, la recherche participative a été fondée sur l'idée de la nécessité d'un rapprochement entre les milieux professionnels et les milieux de recherche, nous le rappelions au début de notre propos. La recherche participative a donc vocation à être lue et étudiée par des non chercheurs. À ce titre, les allant-de-soi que l'on ne précise pas dans des communications de chercheurs pour chercheurs perdent ce statut. La piste ébauchée par Duquette, Lauzon et Saint-Gelais est très intéressante à cet égard, ces derniers situant la plus-value des chercheurs du côté de leurs outils d'analyse.

\section{Savoirs mobilisés}

Dans les travaux constituant ce collectif, les savoirs mobilisés explicitement sont de trois ordres. Ils concernent soit les démarches participatives en recherche, le processus d'enseignement-apprentissage ou les contextes éducatifs.

Une première catégorie de savoirs mobilisés concerne les démarches participatives en recherche. Couture et al. prennent ainsi le temps d'expliquer dans quelles traditions de recherche ils se situent lorsqu'ils développent des équipes-cycles ou des communautés d'apprentissage pour les travaux présentés. Lessard et Coulombe et al. expliquent leurs choix de mettre en place une recherche-action en analysant finement la manière dont cette forme de recherche participative est propice pour rencontrer leurs préoccupations de recherche. Gagnon centre sa contribution sur une forme de collaboration particulière, le 


\section{REVUE HYBRIDE DE L'ÉDUCATION}

groupe de codéveloppement. II est intéressant de remarquer la place occupée par ces références. Elles sont parfois présentées comme des options méthodologiques (par exemple Cody et al. ou Lessard). Parfois, elles sont intégrées non seulement comme option méthodologique, mais aussi comme élément relevant du cadre conceptuel de l'étude (par exemple Couture et al.). Cela peut être lié à des raisons d'ordre rhétorique, mais cela amène tout de même à se poser la question des statuts des connaissances sur les recherches participatives. Ont-elles le même statut épistémologique selon qu'elles servent de cadre conceptuel, de ligne de conduite méthodologique, ou les deux?

Une deuxième catégorie de savoirs mobilisés concerne des savoirs sur le processus d'enseignement/apprentissage. Ces savoirs proviennent de différents champs. Couture et al. mobilisent des savoirs issus de la didactique des sciences pour comprendre les pratiques des enseignants. Cody et al. recourent à des connaissances issues de la recherche sur le recours à des dispositifs d'enseignement de type hybride. Différentes contributions convoquent de manière significative des connaissances sur les élèves. Duquette et al. s'appuient sur la conception scientifique d'une pensée historique pour l'élève. Tardif et Dumoulin s'appuient sur les particularités des élèves présentant une dysphasie. Lessard mobilise des connaissances sur l'importance de la motivation dans le cadre de l'apprentissage de la lecture. Cody et al. s'appuient sur des connaissances qui permettent de comprendre comment des étudiants qui souhaitent devenir enseignants développent leurs compétences professionnelles. Coulombe et al. fondent leur travail sur la reconnaissance des transformations identitaires lorsqu'un professionnel fait le choix de devenir enseignant pour former de nouveaux professionnels. Deux contributions font explicitement référence à des savoirs sur l'enseignement et à des savoirs sur les apprenants : celle de Lessard et celle de Cody et al.

Une troisième catégorie de savoirs mobilisés concerne les connaissances sur les contextes éducatifs. Ces savoirs, qui permettent de 


\section{REVUE HYBRIDE DE L'ÉDUCATION}

mieux cerner le contexte culturel dans lequel se situe la recherche sont de différents ordres dans les contributions. Certaines recherches sont contextualisées en référence aux programmes de formation. Couture et al. et Duquette et al. font ainsi explicitement référence au programme de formation de l'école québécoise. Cody et al. s'appuient sur le référentiel de compétences des enseignants. Amboulé-Abath et al. s'appuient quant à eux sur une description de politique publique qui justifie l'existence même de leur recherche.

Cet essai d'organisation des savoirs mobilisés explicitement dans les articles de ce collectif ouvre des perspectives de recherche intéressantes. Tout d'abord, il conduit à se poser différentes questions, dont les réponses permettraient de mieux comprendre la variété des recherches participatives : comment les savoirs utilisés sont-ils choisis? Ils sont sans doute la plupart du temps apportés par le chercheur, mais sont-ils discutés? La présence ou l'absence d'une telle discussion a sans doute des conséquences sur les résultats de la recherche. Plusieurs contributeurs font référence à l'idée d'une complémentarité des participants, pour le moins dans ce qui concerne les recherches participatives de type collaboratif. Comment cette complémentarité estelle assurée dans les cas où le choix des cadres conceptuels est dévolu au chercheur? Ensuite, notre essai d'organisation permet de construire un paysage à partir des recherches participatives présentées, au sein duquel différents plans se dessinent. Au premier plan de ce paysage, on trouve deux choses : les savoirs issus de la recherche et les savoirs issus de la pratique. Les deux sont intégrés à chacune des contributions. Mais les savoirs issus de la pratique mériteraient d'être davantage explicités. Ils sont évoqués à de nombreuses reprises, mais ils ne sont ni présentés, ni discutés systématiquement, ou en tout cas pas encore au même titre que les savoirs issus de la recherche, malgré un effort fait en ce sens. L'arrière-plan de ce paysage est quant à lui constitué de documents institutionnels, de politiques publiques, autour desquelles chercheurs et acteurs des milieux de pratique semblent se retrouver aisément. Cela est 


\section{$\&$}

\section{REVUE HYBRIDE DE L'ÉDUCATION}

probablement lié au fait que ces textes représentent des cadres de référence partagés donnant des orientations que nul ne peut ignorer. Ces sources permettent donc de faire le pont entre les savoirs issus de la recherche et les savoirs issus de la pratique, et constituent sans doute à ce titre de bonnes portes d'entrée pour avancer dans les questionnements présentés.

\section{Options méthodologiques}

Plusieurs options méthodologiques sont évoquées dans les travaux mis en commun dans ce collectif. Ces options renvoient, d'une part, à la manière de collaborer et, d'autre part, à la nature des données recueillies et des analyses menées.

Pour ce qui concerne les modalités de mise en œuvre des recherches participatives, il est intéressant de constater que la question de la transformation des pratiques par la voie de la collaboration a interpellé des équipes s'appuyant sur le modèle de la recherche collaborative (Couture et al.; Amboulé-Abath et al.; Duquette et al.) et de la recherche-action (Lessard; Coulombe et al.) et même dans des approches compréhensives visant à décrire une situation afin d'envisager des pistes à explorer et à développer avec différents acteurs (Tardif et Dumoulin; Cody et al.; Gagnon). Cette diversité témoigne de la variété des voies de collaboration pour transformer les pratiques utilisées dans la région du Saguenay-Lac-St-Jean. Les manière de faire, à l'intérieur de chacune de ces manières de collaborer sont également variées. Par exemple, pour ce qui concerne les recherches collaboratives, certains mettent en place des équipes-cycles ou école, des communautés d'apprentissage ou de recherche, des groupes de codéveloppement, modalités qui reviennent aussi dans les recherches-actions. Ces modalités ne sont donc pas spécifiques à un type de recherche en particulier. Elles font partie d'un ensemble de possibilités à exploiter dans le cadre de la recherche participative. 


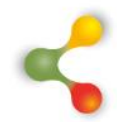

\section{REVUE HYBRIDE DE L'ÉDUCATION}

Les articles de ce collectif présentent également une grande richesse au plan des données recueillies et des analyses mises en place. Certains se situent dans une tradition qualitative (Couture et al.; Coulombe et al.) et basent leur collecte de données sur des outils comme l'observation qualitative, l'entretien qualitatif. En général, les données semblent être analysées via une démarche inductive modérée, même si cela n'est pas toujours explicite. D'autres se situent dans une démarche mixte (Duquette et al.; Lessard; Cody et al.). Dans ces cas, les outils qualitatifs évoqués cidessus se voient accompagnés, le plus souvent, de questionnaires. Dans toutes ces contributions, le volet quantitatif sert à établir un portrait global du contexte de réalisation de la recherche. Enfin, une contribution s'appuie sur une méthodologie quantitative (Amboulé-Abath et al.). Là encore, cela témoigne de la richesse des recherches mises en œuvre. Cela montre aussi la vitalité des recherches participatives recensées. En effet, traditionnellement, les recherches participatives en éducation, au Québec, s'inscrivent plutôt dans un paradigme qualitatif. Ce collectif témoigne du fait que le recours à des outils quantitatifs, en plus d'outils qualitatifs, peut donner des résultats intéressants pour mieux cerner les contextes dans lesquels les recherches s'ancrent.

\section{Résultats et retombées}

En termes de résultats, la diversité témoigne encore de la richesse de cette mise en commun. En effet, qu'il s'agisse d'exemples de pratique en science et technologie (Couture et al.), de portraits de comportements de parents et du point de vue de leurs adolescents dysphasiques (Tardif et Dumoulin), de difficultés d'élèves et de stratégies didactiques pour favoriser la réussite de l'épreuve unique d'histoire (Duquette et al.), de pistes d'intervention pour intégrer la lecture à un projet de plein-air (Lessard), des avantages et des défis de la collaboration pour évaluer un projet d'établissement (Amboulé-Abath et al.) ou pour soutenir les nouveaux enseignants en formation professionnelle (Coulombe et al.), ainsi que de dispositifs d'accompagnement (Cody et al.), ces résultats sont tous porteurs de 


\section{REVUE HYBRIDE DE L'ÉDUCATION}

nouveaux savoirs. Sans nécessairement être explicitement identifiés, ces savoirs concernent soit les résultats de la collaboration, en termes de stratégies et de pistes d'intervention, soit la collaboration en elle-même, en termes d'avantages et de défis.

Une analyse encore plus fine des résultats présentés dans les recherches de ce collectif permettrait de voir avec précision sur quelles dimensions de l'intervention éducative ou de la gestion organisationnelle chercheurs et praticiens travaillent ensemble. En ce qui concerne les stratégies et les pistes d'intervention, les réflexions menées conjointement portent-elles davantage sur les contenus, en lien avec les programmes, les façons de les enseigner, la gestion de la classe ou toute autre question d'ordres didactique et pédagogique? Sur quels aspects plus particuliers les pratiques se transforment-elles? De quels ordres sont les avantages et les défis de la collaboration pour soutenir les choix institutionnels ou le développement professionnel? Ces avantages et ces défis relèvent-ils plus des organisations ou des personnes? Ce sont là autant de questions que nous pouvons poser pour essayer de mieux saisir la nature des savoirs qui se construisent collectivement et les ressources qu'ils mobilisent et desservent à la fois. Dans un projet collectif, il serait intéressant de se donner un cadre commun afin de faire cette analyse pour documenter avec plus de précision la nature des savoirs qui se construisent dans le cadre de recherches participatives.

Notons par ailleurs que la satisfaction exprimée par les acteurs engagés dans les travaux de recherche participative semble unanime. Le temps de réflexion et le partage d'expertise sont évoqués à maintes reprises comme éléments forts à retenir des contributions de ce collectif, mais également de contraintes en termes de ressources mobilisées. Cette satisfaction nous amène à poser la question des suites à donner à de tels projets. La principale question qui se pose est de savoir comment en faire bénéficier un plus grand nombre d'acteurs. Considérant l'investissement très important que réclame ce genre d'approche, cette question semble cruciale. Mais elle n'a pour le moment pas vraiment de réponse. Malgré 


\section{REVUE HYBRIDE DE L'ÉDUCATION}

cette réserve, une analyse encore plus fine des transformations de pratique et du processus de collaboration qui s'opèrent dans le cadre de recherches participatives aiderait peut-être à envisager des modalités d'appropriation réalistes pour des acteurs n'ayant pas participé à ces projets. C'est toute la question de la diffusion et du transfert qui se pose ici.

\section{Production et diffusion}

Le format de présentation de ce collectif ne permet pas de voir comment les résultats des travaux sont diffusés dans les milieux de recherche et de pratique. II permet d'en avoir un aperçu selon les normes de la recherche mais cache peut-être des initiatives locales ou des réinvestissements dont nous témoignons rarement. À ce chapitre, pensons aux outils ou pistes de développement repris par différents acteurs, aux réflexions collectives partagées dans le milieu scolaire ou même dans les activités de formation des enseignants. II y a là tout un pan de retombées difficiles à documenter. D'ailleurs, nous constatons qu'il y a bien, en cours de processus, diffusion des résultats d'analyse au sein des équipes qui travaillent ensemble puisque des décisions et des pistes de développement émanent de ces analyses. C'est au-delà de ces équipes qu'il est plus difficile de voir des retombées pour la pratique. Pour la recherche, les publications et les communications permettent de voir les retombées en termes de production de connaissances, mais pour la pratique, des modes de diffusion permettant que d'autres acteurs s'approprient et bénéficient des résultats de ces recherches restent à construire. Pensons entre autres au travail de réinvestissement qui pourrait se faire si des chercheurs travaillaient, avec des conseillers pédagogiques, à partager le fruit de ces recherches dans de nouvelles réflexions engagées avec d'autres enseignants. Cela exigerait de réfléchir à nos structures mais nous permettrait d'innover à la fois au regard des contenus et des processus de formation continue et de développement professionnel. II y a peut-être là un défi à relever collectivement, considérant que la recherche participative peut « contribuer concrètement 


\section{REVUE HYBRIDE DE L'ÉDUCATION}

et collectivement à l'enrichissement de la profession enseignante » tel que recommandé par le Conseil supérieur de l'éducation (2014).

\section{Pour conclure}

À la lumière de cette analyse, retenons que la recherche participative contribue à la transformation des pratiques dans différents contextes et sous différents angles. Que ce soit en contexte de classe, de relation avec les familles, de gestion organisationnelle, de supervision de stage ou d'insertion professionnelle, les recherches présentées dans ce collectif offrent de multiples possibilités pour travailler ensemble au développement de la profession enseignante. En termes de résultats, les pistes d'intervention, les avantages et les défis de la collaboration montrent bien qu'il y a des retombées pour la recherche et pour la pratique, mais ces retombées demeurent assez vagues et mériteraient d'être précisées pour un meilleur réinvestissement dans d'autres contextes.

La diversité de possibilités qu'offre la recherche participative invite à la réflexion afin de caractériser un peu plus précisément ce qui se construit collectivement. Pour ce faire, nous suggérons, comme pistes de développement, de documenter plus en détail : les rôles des acteurs qui s'engagent dans des recherches participatives; les processus de collaboration qui s'y opèrent ainsi que les savoirs construits collectivement. Les retombées pour la recherche étant plus faciles à saisir, nous suggérons aussi de regarder de plus près ce qui peut être réinvesti de ces recherches en contexte de pratique, non seulement en termes de pistes d'intervention, mais aussi de modalités pour soutenir le développement et l'enrichissement de la profession enseignante. C'est un projet commun auquel nous pouvons tous contribuer selon des perspectives qui gagnent à être mises en commun, ce dont témoignent les exemples de recherches participatives faisant l'objet de cette publication. Retenons pour conclure que malgré les défis que présente le travail réalisé en collaboration, il contribue à changer le regard des uns et 


\section{REVUE HYBRIDE DE L'ÉDUCATION}

des autres, qu'ils soient praticiens ou chercheurs, réduisant ainsi l'écart entre la pratique et la recherche, et ce, au profit des élèves.

\section{Références}

Anadón, M. et Savoie-Zajc, L. (2007). La recherche-action dans certains pays anglo-saxons et latino-américains. Une forme de recherche participative. Dans $M$. Anadón (dir.), La recherche participative (p. 11-30). Québec, QC : PUQ.

Conseil supérieur de l'éducation (2014). Le développement professionnel, un enrichissement pour toute la profession enseignante. Québec, QC : Publications du gouvernement du Québec.

Desgagné, S. (1998). La position du chercheur en recherche collaborative : illustration de médiation entre culture universitaire et culture scolaire. Recherches qualitatives, 18, 77-105.

Desgagné, S., Bednarz, N., Couture, C., Poirier, L. et Lebuis, P. (2001). L'approche collaborative de recherche en éducation: un nouveau rapport à établir entre recherche et formation. Revue des Sciences de l'Éducation, XXVII(1), 33-64.

Lafortune, L. (2008). Compétences professionnelles pour l'accompagnement d'un changement. Québec, QC : Presses de l'Université du Québec.

Marcel, J.-F., Dupriez, V. et Bagnoud, D.P. (2007). Le métier d'enseignant : nouvelles pratiques, nouvelles recherches. Dans J.-F. Marcel, V. Dupriez, D.P. Bagnoud, et M. Tardif (dir), Coordonner, collaborer, coopérer (p.7-20). Bruxelles : De Boeck Université.

Savoie-Zajc, L. (2005). Soutenir l'émergence de communauté d'apprentissage au sein de communautés de pratique ou les défis de l'accompagnement au changement. Dans L. Sauvé, I. Orellana et É. Van Steenberghe (dir.), Éducation et environnement : un 


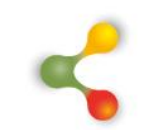

\section{REVUE HYBRIDE DE L'ÉDUCATION}

croisement de savoirs (p. 63-75). Montréal, QC : Les cahiers scientifiques de l'ACFAS. 